\title{
Design and Investigation of Circularly Polarized RMPA with Chiral Metamaterial Cover
}

\author{
Preet kaur $^{1 *}$, S.K.Aggarwal ${ }^{2}$, Asok $^{3} e^{3}$ \\ ${ }^{1,2}$ Electronics Department, YMCA university of Science and Technology Faridabad, India \\ ${ }^{3}$ Director, National Institute of Technology, Patna(Bihar)
}

\begin{abstract}
In this paper, a simple method for designing a circular polarized antenna using chiral metamaterial is presented. The antenna proposed in this paper consists of conventional linear polarized Rectangular Microstrip Patch Antenna (RMPA) and cover of bilayer twisted cross wire Chiral Metamaterial (CM). The placement of CM cover over the linearly polarized RMPA changes its polarization from linear to circular and the antenna produces a left handed circularly polarized wave at resonant frequency of $6.24 \mathrm{GHz}$ with axial ratio of $0.46 \mathrm{~dB}$. Investigation of performance parameter of proposed antenna and parameters of unit cell of CM is also presented in the paper.
\end{abstract}

Index Terms: Chiral Metamaterial (CM), Left hand Circularly Polarized(LCP), Right hand Circular Polarized(RCP), Rectangular Microstrip Patch antenna(RMPA),linear polarization(LP), circular polarization $(\mathrm{CP})$

(C) 2016 Published by MECS Publisher. Selection and/or peer review under responsibility of the Research Association of Modern Education and Computer Science

\section{Introduction}

Circularly polarized antennas have gained much attention in wireless communication, radar systems and satellite communications as these antennas are more reliable as compared to linearly polarized (LP) antennas. The waves produced by $\mathrm{CP}$ antennas have better mobility, penetration and less vulnerable to harsh weather conditions as compared to linear waves. Other advantages of CP antennas are elimination of alignment issues, better connectivity, reduced multipath effects and better signal strength through obstacle [1-2]. Circular polarized antennas can be designed in different shapes and orientation like monopole antenna[3], helix antenna, horn antenna, but CP Microstrip patch antennas [4-7] are more popular in today's rapid developing scenario of electronics and wireless industry because of small size, low cost, light weight, low profile, easily mountable

* Corresponding author.

E-mail address: preetmoar@gmail.com 
and can be easily integrated with other planer components.

Several techniques have been reported in literature to design a patch antenna with CP [8-11]. All of these techniques use the complex feeding mechanism to produce two degenerate orthogonal modes of equal amplitude and $90^{\circ}$ phase shift. In Conventional designing methods as reported above, feeding mechanism makes the design and fabrication of CP antenna difficult.

Recently, researchers have proposed the use of chiral metamaterial for designing the CP antenna [12-14]. This technique exploits the unique inherent properties of chiral metamaterial to produce $\mathrm{CP}$ and eliminates the use of complex feeding mechanism as used in conventional techniques.

Chiral metamaterial [15] are the metamaterial [16-19] which lacks the mirror symmetry in any plane. This property results in breaking the degeneracy between the two CP waves, Left hand circularly polarized wave and right handed circularly polarized wave. Due to this property, when the LP wave which can be assumed of composed of opposite circularly polarized wave impinges on Chiral metamaterial, it generate two possible effects in LP wave: Circular dichroism and optical activity. Optical activity means the rotation of polarization plane of wave and circular dichroism means change of polarization from linear to elliptical. The circular dichroism and optical activity effect of chiral metamaterial can be exploited to change polarization of RMPA from linear to circular. Chiral metamaterial with Y shape [20], twisted and nested U shape [21-22], wheel shaped[23], fractal shaped[24] and spiral shape[25-26] etc. are proposed in literature .

In this paper, Bilayer twisted cross wire chiral metamaterial (CM) is used which is published in [27]. Zarifi. et. el. [28] has used this CM to improve the performance of circularly polarized antenna, but in this paper, it is used to change the polarization of rectangular patch antenna from linear to circular. All the performance parameters of antenna like antenna transmission, axial ratio, radiation pattern, and gain are thoroughly investigated and parameters of unit cell of $\mathrm{CM}$ are also studied.

\section{Design of Reference RMPA Antenna}

Reference RMPA antenna is designed at resonant frequency of $6.2 \mathrm{GHz}$ using equation (1) [1] on Fr4 epoxy substrate having dielectric constant $\left(\varepsilon_{\mathrm{r}}\right)=4.4$ and loss tangent $(\delta)=0.0025$.

$$
L=\frac{c}{2 f_{o} \sqrt{\varepsilon_{\text {reff }}}}
$$

Where $f_{o}=$ resonant frequency, $\mathrm{L}=$ length of the rectangular shaped patch, $\mathrm{c}=$ is velocity of light, $\varepsilon_{\mathrm{r}}=$ relative permittivity of the substrate and $\varepsilon_{\text {reff }}=$ effective dielectric constant of substrate, which is calculated using (2)

$$
\varepsilon_{\text {reff }}=\frac{\left(\varepsilon_{r}+1\right)}{2}+\frac{\left(\varepsilon_{r}-1\right)}{2}\left(1+\frac{12 h}{W}\right)^{-1 / 2}
$$

$\mathrm{W}=$ width of patch antenna and is calculated using equation (3)

$$
W=\frac{c}{2 f_{o} \sqrt{\frac{\left(\varepsilon_{r}+1\right)}{2}}}
$$

The designed reference rectangular patch antenna is simulated and optimized using HFSS as shown in Fig 1. The patch antenna has length $(\mathrm{L})=10.02 \mathrm{~mm}$ and width $(\mathrm{W})=14.72 \mathrm{~mm}$. The ground size dimensions are 75 $\mathrm{mm} \times 75 \mathrm{~mm}$ and is fed by coaxial probe situated at $(35.4 \mathrm{~mm}, 39.22 \mathrm{~mm})$ in Cartesian coordinates.

Fig 2. depicts the reflection coefficient of RMPA, it resonates at $6.24 \mathrm{GHz}$ with reflection coefficient of -25 $\mathrm{dB}$. The reference has the axial ratio of $41 \mathrm{~dB}$ at resonant frequency as shown in Fig 3 .Fig. 4 represents the LCP and RCP gain of reference antenna, which is same for both modes. From axial ratio and gain graph it is 
clear that reference RMPA is linearly polarized.

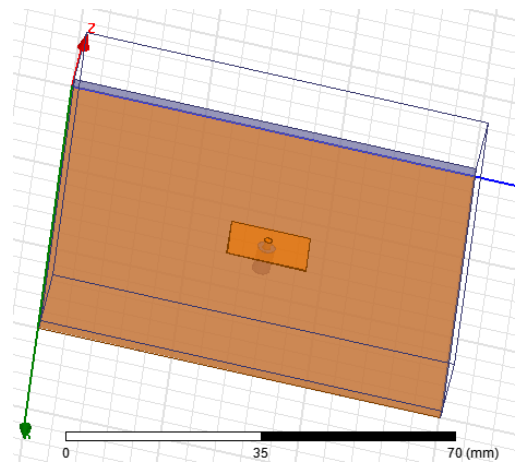

Fig.1. Simulated Model of reference RMPA fed by coaxial feed

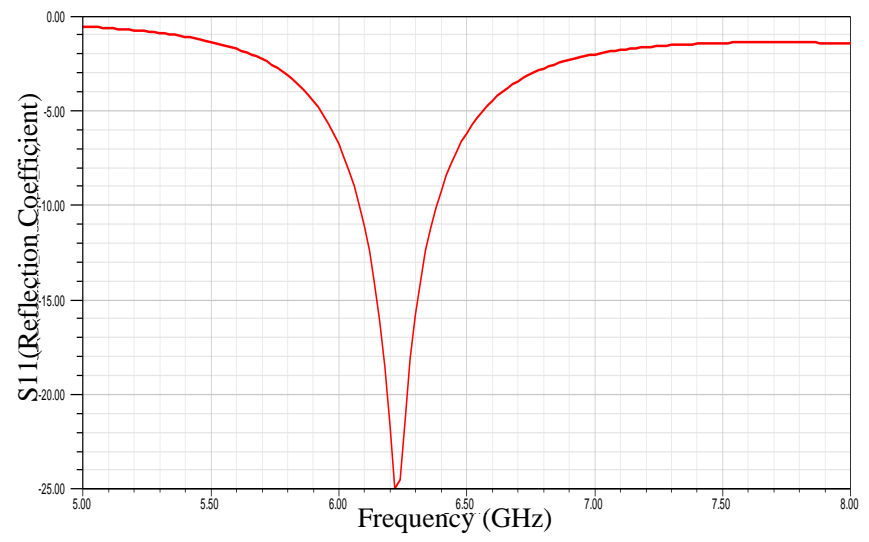

Fig.2. Simulated reflection coefficient (S11) of reference RMPA

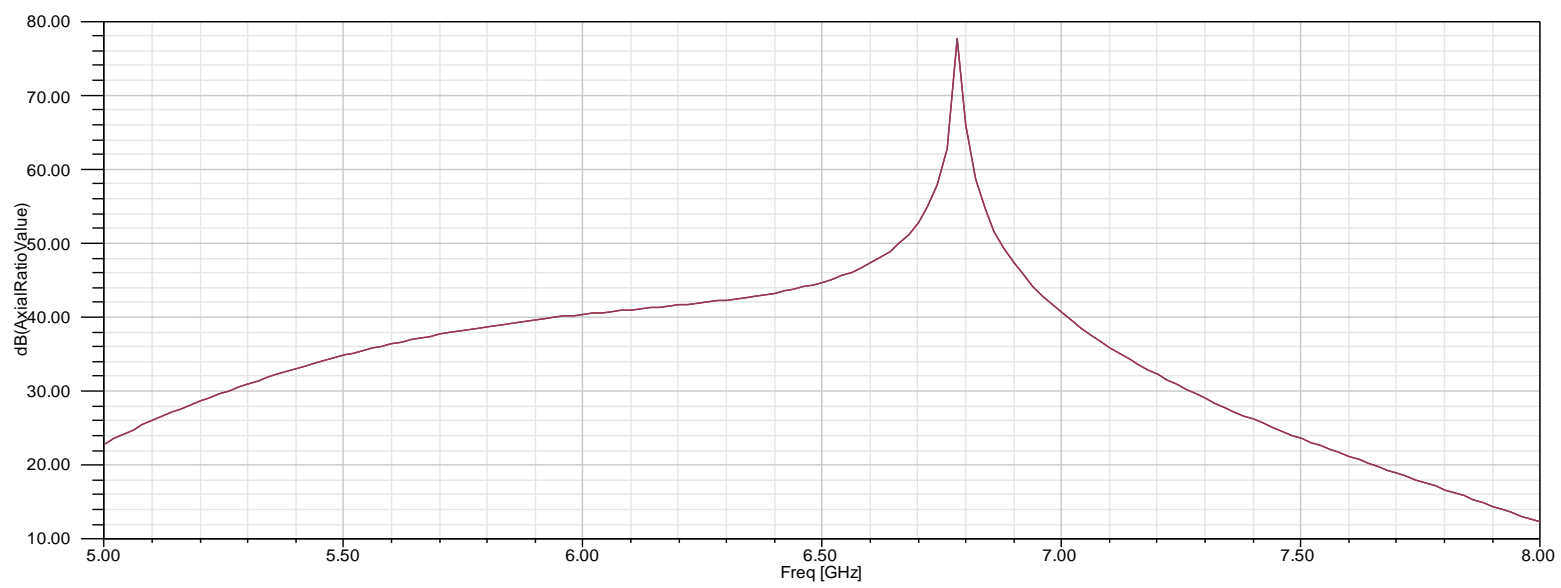

Fig.3. Axial ratio of reference RMPA 


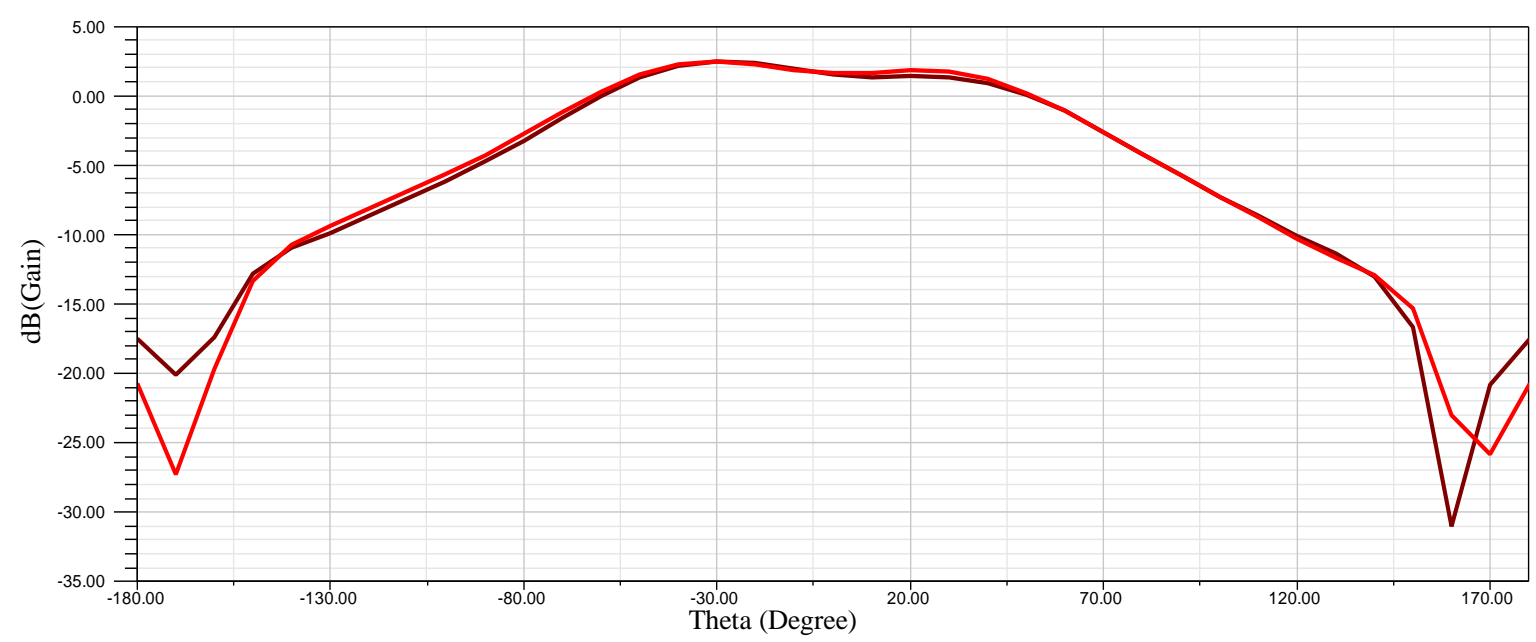

Fig.4. Gain of reference RMPA

\section{Design and Investigation of Unit Cell of Chiral Metamaterial}

In this paper, bilayer twisted cross wire chiral metamaterial (CM) [25] is used. Unit cell of CM consist of twisted cross wires printed on both side of Fr4 substrate having $\varepsilon_{\mathrm{r}}=4.4$ and loss tangent $(\delta)=0.0025$ and thickness $1.6 \mathrm{~mm}$. The length and width of twisted cross wires are $14 \mathrm{~mm}$ and $1 \mathrm{~mm}$ respectively as shown in Fig. 5 and Fig 6.

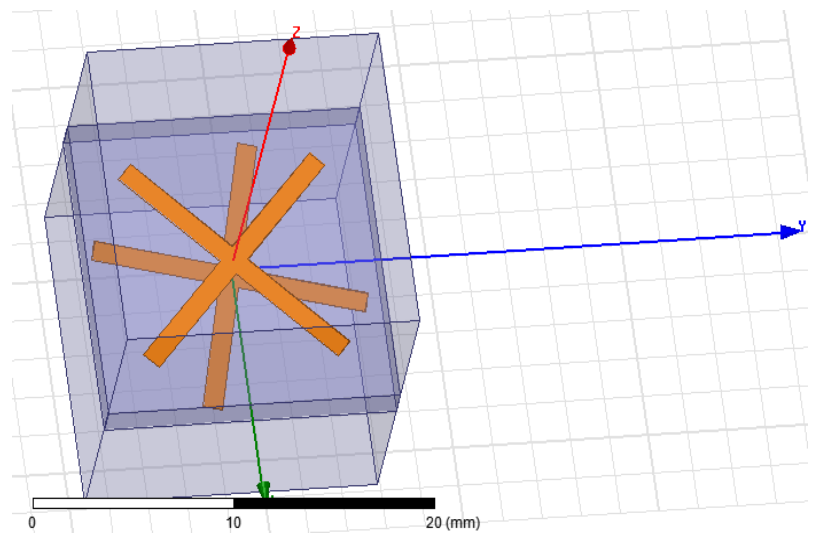

Fig.5. Unit Cell simulation model of Bilayer twisted cross wire chiral metamaterial in HFSS 


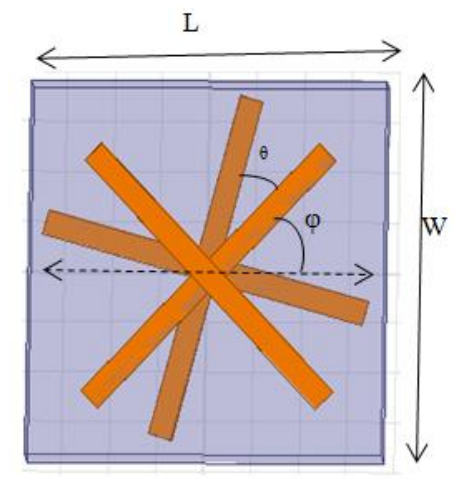

Fig.6. Bilayer twisted cross wire $\mathrm{CM}$ with geometric parameters $\mathrm{L}=15 \mathrm{~mm}, \mathrm{~W}=15 \mathrm{~mm}, \theta=15^{0}, \varphi=45^{0}$

For simulation, unit cell boundaries are applied to $\mathrm{x}$ and $\mathrm{y}$ direction and absorbing boundary conditions are applied to $\mathrm{z}$ axis. In simulation, linearly polarized (LP) wave is allowed to incident on the CM. As the LP is a combination of LCP and RCP.

$$
L P=\frac{1}{2} L C P+\frac{1}{2} R C P
$$

To achieve good CP wave, either LCP or RCP wave need to be small. Two Eigen modes of electromagnetic wave is two circularly polarized wave i.e. left hand circularly polarized wave (LCP) and right hand circularly polarized (RCP) wave in CM. The four circular transmission coefficients $T_{++}, T_{+-}, T_{-+}, T_{--}$which describe the response of chiral metamaterial can be expressed in terms of four linear coefficients $\mathrm{T}_{\mathrm{xx}}, \mathrm{T}_{\mathrm{xy}}, \mathrm{T}_{\mathrm{yx}}, \mathrm{T}_{\mathrm{yy}}$ by following transformation[28].

$$
\left(\begin{array}{ll}
T_{++} & T_{+-} \\
T_{-+} & T_{--}
\end{array}\right)=\left(\begin{array}{ll}
\left(T_{x x}+T_{y y}\right)-i\left(T_{x y}-T_{y x}\right) & \left(T_{x x}-T_{y y}\right)+i\left(T_{x y}+T_{y x}\right) \\
\left(T_{x x}-T_{y y}\right)-i\left(T_{x y}+T_{y x}\right) & \left(T_{x x}+T_{y y}\right)-i\left(T_{x y}+T_{y x}\right)
\end{array}\right)
$$

Where the first subscript shows the transmitted field polarization and second subscript shows incident field polarization.

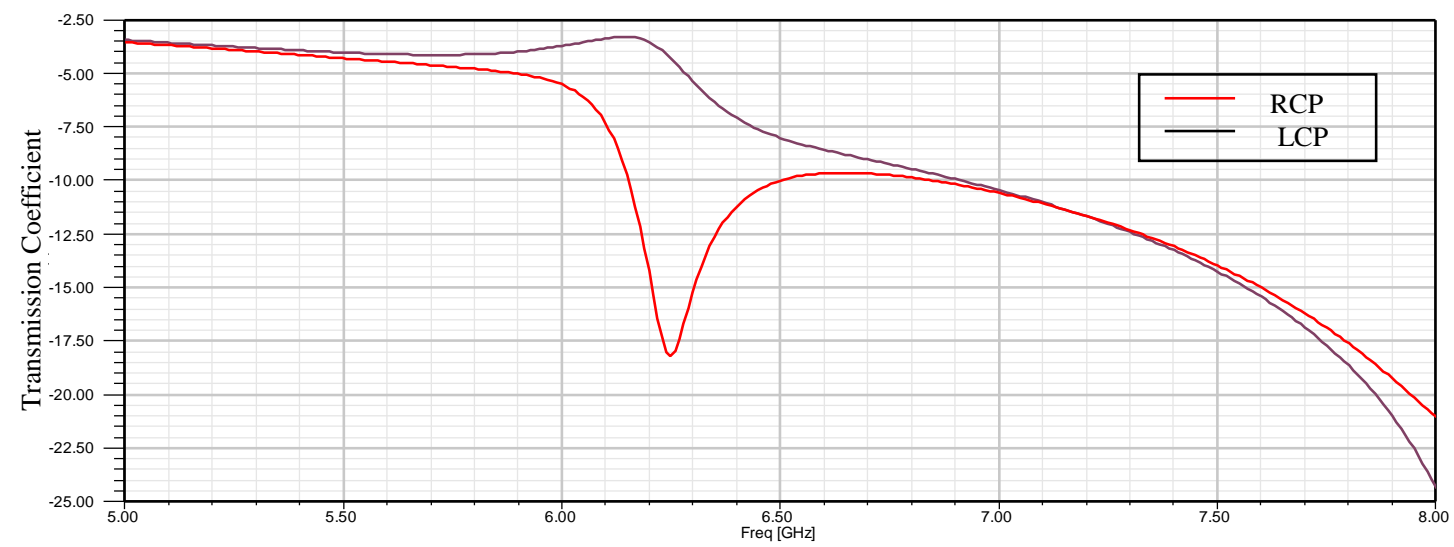

Fig.7. Transmission coefficient of LCP and RCP Wave in chiral metamaterial 
Fig. 7 shows the transmission coefficient of RCP and LCP wave in chiral metamaterial. The CM is designed at resonant frequency of reference patch antenna at $6.24 \mathrm{GHz}$. In this frequency range it offer different media to both the waves, LCP wave is allowed to pass through the chiral metamaterial and RCP wave is suppressed. It offers different refractive index to LCP and RCP waves and due to this polarization of the wave after passing through chiral metamaterial changes from Linear Polarization to left hand Circular Polarization.

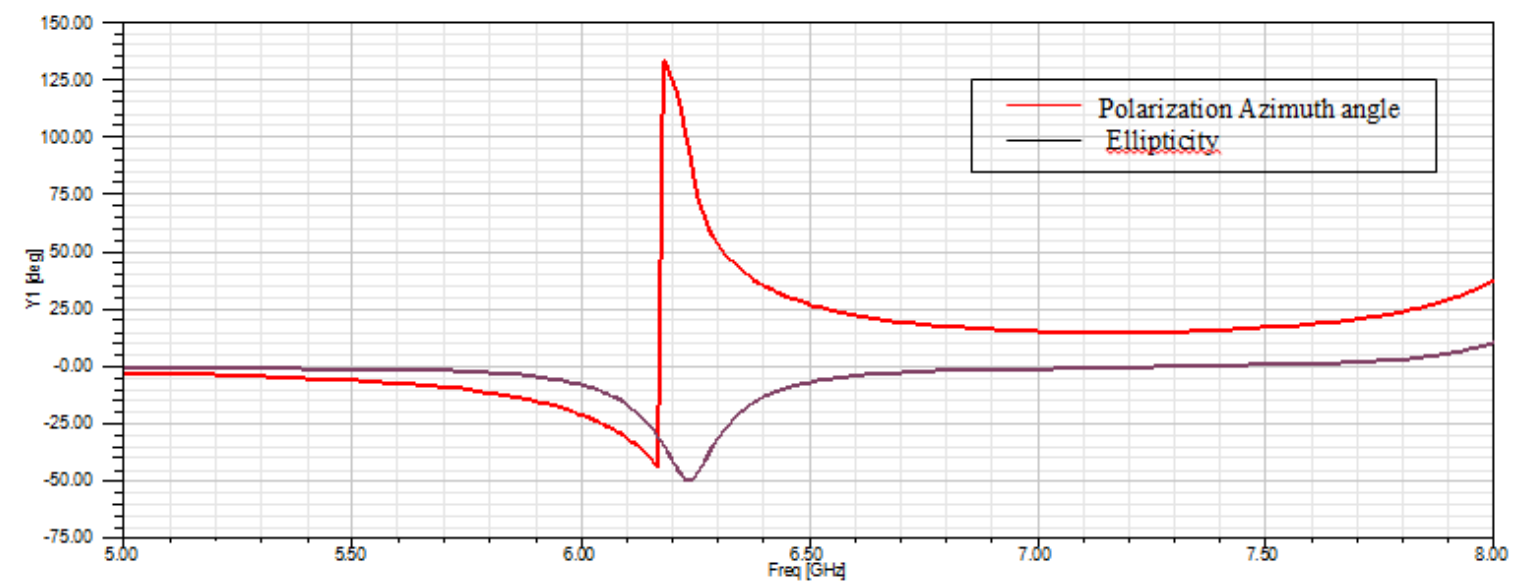

Fig.8. Ellipticity and Azimuth rotation angle of Electromagnetic wave after passing through chiral metamaterial

Difference between amplitude and phase of RCP and LCP transmission coefficient is characterized by ellipticity $\eta$ and azimuth rotation angle $\theta$ and can be expressed in terms of transmission coefficients as given in equation (6) and (7).

$$
\begin{aligned}
& \eta=\arctan \left(\frac{\left|T_{++}\right|-\left|T_{--}\right|}{\left|T_{++}\right|+\left|T_{--}\right|}\right) \\
& \theta=\frac{1}{2}\left[\arg \left(T_{++}\right)-\arg \left(T_{--}\right)\right]
\end{aligned}
$$

Fig. 8, shows the retrieved ellipticity and azimuth rotation angle of EM wave using equation 5 and 6 . The resultant wave from CM has ellipticity $45^{\circ}$ and azimuth rotation angle is $90^{\circ}$, which depict the resulting wave is circularly polarized.

\section{Design and Investigation of Proposed CP RMPA}

After designing the twisted cross unit cell of chiral metamaterial, a $5 \times 5$ array of unit cells is applied over the RMPA at a spacing $d$ as shown in Fig. 9.Numerical simulation is performed using HFSS in the range of $4 \mathrm{GHz}$ to $6 \mathrm{GHz}$ shows that optimum value of the spacing between the RMPA and CM cover is $\mathrm{d}=20 \mathrm{~mm}$. The dimension of $\mathrm{CM}$ cover consisting of $5 \times 5$ unit cells is same as the substrate size of RMPA. 


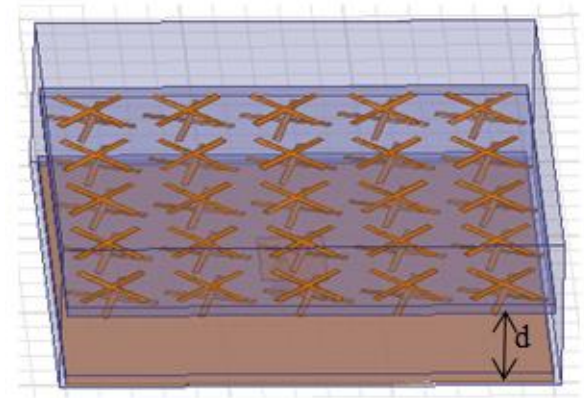

Fig.9. Proposed CP coaxial fed RMPA with chiral Metamaterial cover

Fig. 10 shows the reflection coefficient of RMPA antenna with CM cover. It can be seen from this graph that after applying CM cover over the patch antenna reflection coefficient improves slightly as compared to reference antenna and resonant frequency remains the same, $6.24 \mathrm{GHz}$.

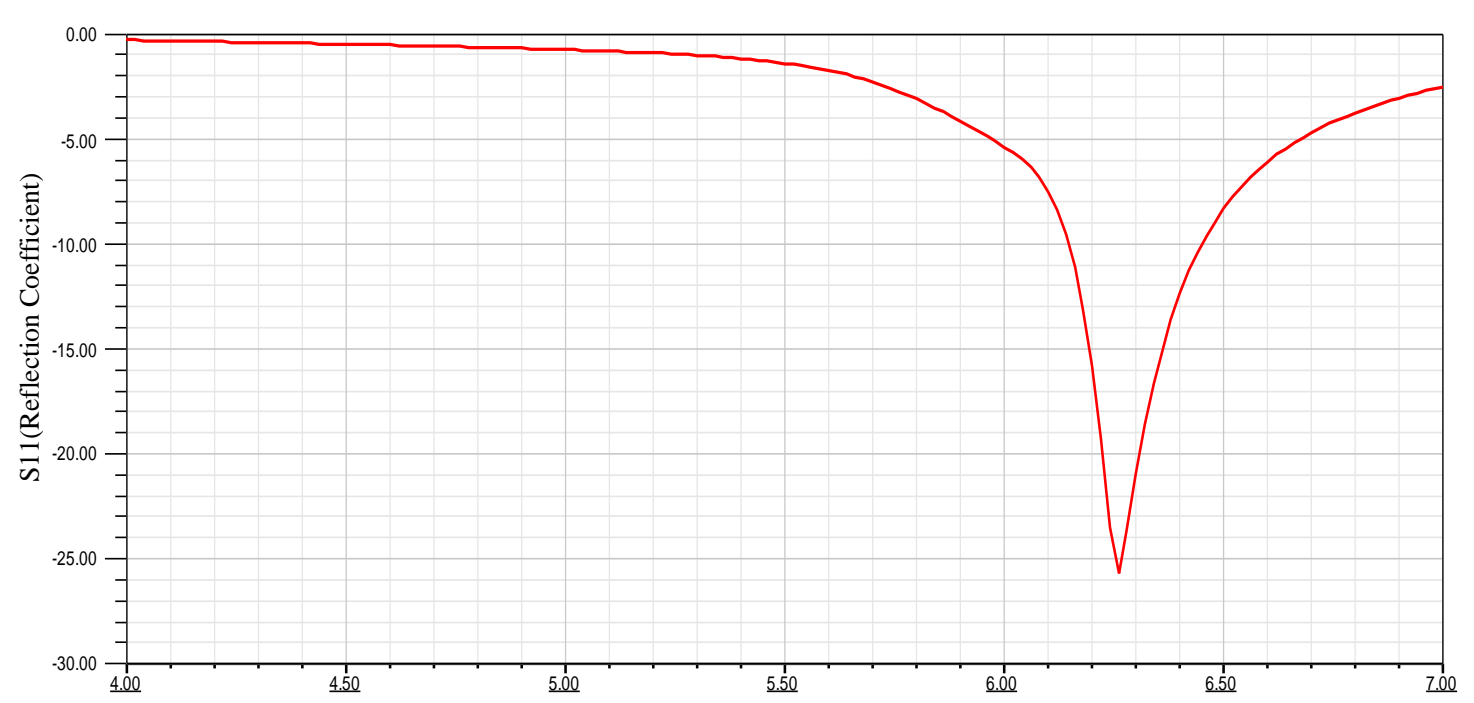

Fig.10. Reflection coefficients (S11) of RMPA with CM cover

Fig. 11 gives the rectangular patch antenna axial ratio, which depicts that $3 \mathrm{~dB}$ axial-ratio bandwidth, covers from $6.16 \mathrm{GHz}$ to $6.40 \mathrm{GHz}$. The effect of applying the $\mathrm{CM}$ cover over the patch antenna changes its axial ratio $41 \mathrm{~dB}$ to $0.46 \mathrm{~dB}$ at resonant frequency of $6.24 \mathrm{GHz}$.

Thus the antenna polarization changes from linear to circular due to CM cover. Fig. 12 depicts the LCP and RCP gain of proposed antenna. In reference antenna both LCP and RCP gain are approximately same as shown in Fig. 4 and hence the antenna is LP. But the application of CM over patch antenna excites the LCP and supress the RCP as can be seen from graph shown in Fig. 12. Thus antenna polarization mode changes from LP to $\mathrm{CP}$ and LCP mode excites in proposed antenna. 


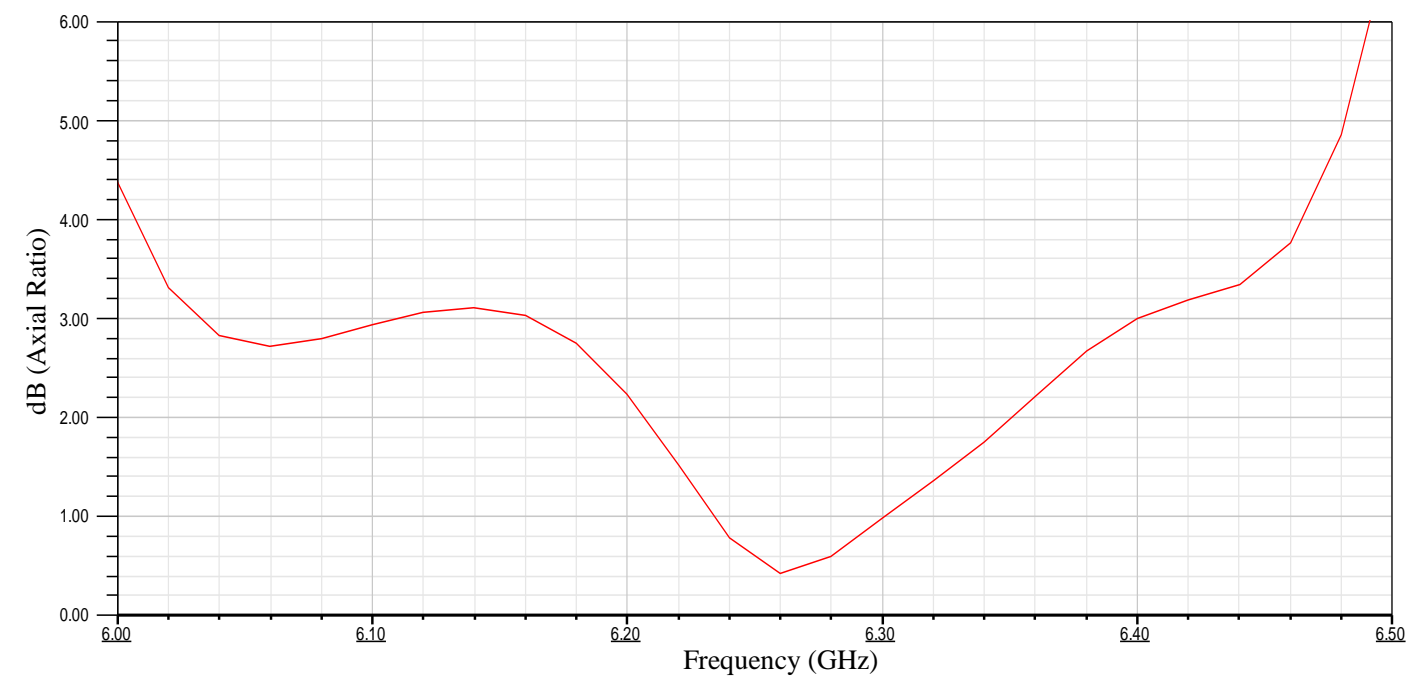

Fig.11. Axial Ratio of RMPA with CM cover

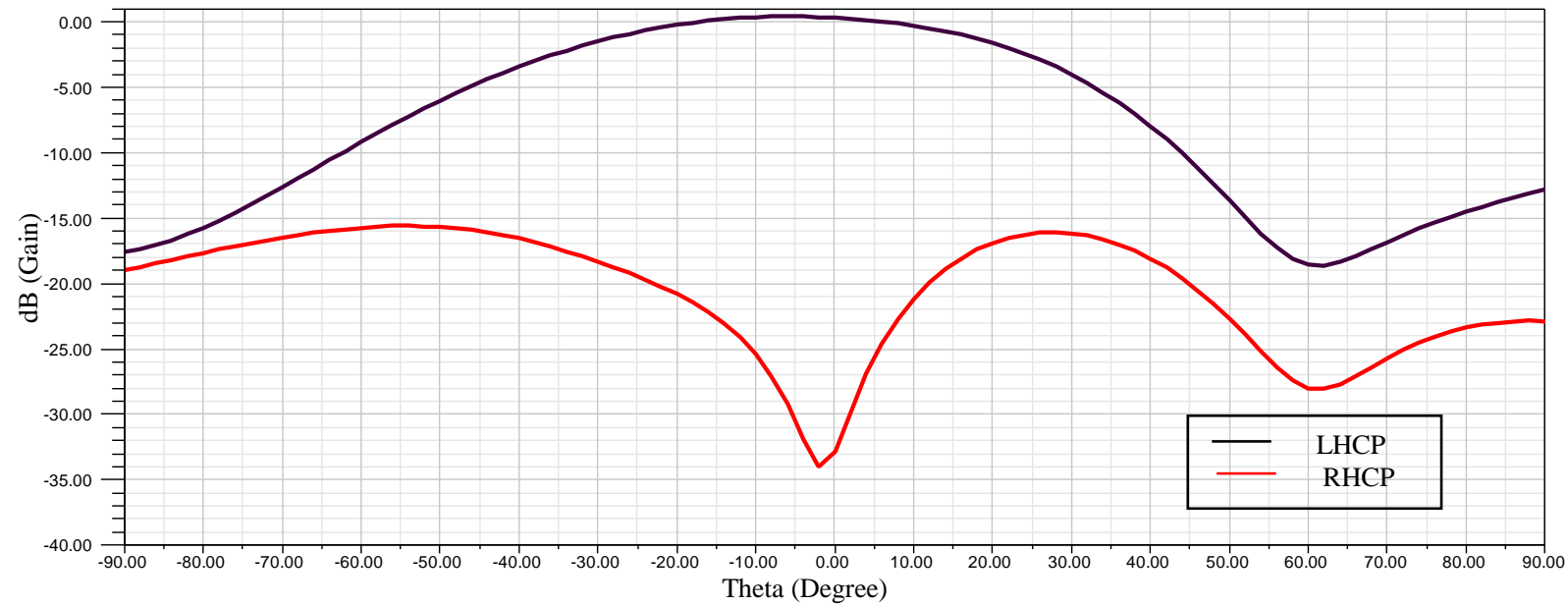

Fig.12. Gain of RMPA with CM cover

\section{Conclusions}

In this paper, $\mathrm{CP}$ patch antenna based on chiral metamaterial is proposed. The proposed CP patch antenna consists of traditional rectangular patch antenna and twisted cross wire CM. Investigation and results shows that Conventional LP RMPA changed to CP patch antenna, when CM cover is placed over it and this CM based antenna radiates the LCP wave at $6.24 \mathrm{GHz}$ with the $3 \mathrm{~dB}$ axial ratio bandwidth covers from $6.16 \mathrm{GHz}$ to 6.40 $\mathrm{GHz}$. The designed antenna has advantage of simple structure, low cost and it is expected to benefit the application in wireless and satellite communication. Future work will focus on gain enhancement of CP antenna as the proposed antenna gain is less than reference antenna. 


\section{References}

[1] K. L. Wong, Compact and Broadband Microstrip Antennas. New York: Wiley, 2002.

[2] R. Garg, P. Bhartia, I. Bahl, and A. Ittipiboon, Microstrip Antenna Design Handbook. Norwood, MA: Artech House, 2001.

[3] Christina F. Jou, Jin-Wei Wu, Chien-Jen Wang, "Novel Broadband Monopole Antennas With Dual-Band Circular Polarization”, IEEE Trans. Antennas Propagat., VOL. 57, pp. 1027-1034, 2009

[4] M. Barakat, C. Delaveaud, F. Ndagijimana, "Circularly Polarized Antenna on SOI for the 60 ghz band", EuCAP, 2007.

[5] Wen-Shyang Chen, Chun-KunWu, Kin-LuWong, "Novel Compact Circularly Polarized Square microstrip Antenna”, IEEE Trans. Antennas Propagat., VOL. 49, pp. 340-342, 2001.

[6] Shyh-Yeong Ke, "Single-feed Microstrip Antenna with Two-layer Substrate for Circular Polarization", ISCOM, 2005

[7] Lei Bian, Yong-Xin Guo, L. C. Ong, Xiang-Quanb Shi, "Wideband Circularly-Polarized Patch Antenna", IEEE Trans. Antennas Propagat., VOL.54, pp. 2682-2686, 2006.

[8] X.L.Bao and M.J.Ammann, "Comparision of several novel annular ring microstrip patch antennas for circular polarization," Journal of Electromagnetic Waves Appl., vol. 20, no. 11, pp. 1427-1438, 2006.

[9] K.F. Tong and J.J.Huang, "New proximity coupled feeding method for reconfigurable circularly polarized microstrip ring antennas," IEEE Trans. Antennas Propag., vol. 56,no.7,pp. 1860-1866, jul.2008.

[10] K.M. Mak and K.M.Luk, "A shorted cross bowtie patch antenns with a cross dipole for circular polarization,” in proc. IEEE Antennas Propag. Int. Symp. 2007, pp. 2702-2705.

[11] Y.F.Lin, H.M. Chen and S.C.Lin, "A new coupling mechanism for circularly polarized annular-ring patch antenna,” IEEE Trans. Antenna Propag. Vol. 56, no. 305-308, 2013.

[12] M.Malathong, A. Sonsilphong, W.Panradit and N. Wongkasem, "Chiral Metamatrial based circularly polarized microstrip antennas," IEEE APWC, 2011, pp. 898-901.

[13] Xiaoliang Ma, Cheng Huang, Wenbo Pan, Bo Zhao, Jianhua Cui and Xiangang Loo, "A Dual Circularly Polarized Horn Antenna in Ku-band Based on Chiral Metamaterial," IEEE Transaction on Antennas and Propagation, Vol. 62, No. 4, pp. 2307-2310, April 2014.

[14] Yahong Liu, Kun Sang, Ying Qi, Shuai Gu and Xiaopeng Zhao, “ Investigation of Circularly polarized patch Antenna with Chiral Metamaterial", IEEE antennas and Wireless propagation letters, vol.12, pp. 1359-1362, 2013.

[15] Rongkuo Zhao, Thomas Koschny and Costas M. Soukoulis, "Chiral metamaterials: retrieval of the effective parameters with and without substrate," OPTICS EXPRESS, Vol. 18, No. 14, June 2010.

[16] Preet Kaur, S. K. aggarwal and Asok De, " Design of compact rectangular patch antenna using square grid and I shaped metamaterial" , IEEE International conference on signal processing and communication(ICSC), March 2015

[17] Ammar Nadal Shareef, Amer Basim Shaalanb, “Fractal Peano Antenna Covered by Two Layers of Modified Ring Resonator," International Journal of Wireless and Microwave Technologies(IJWMT), 2015, 2, 1-11d Microwave Technologies, vol.2, pp. 1-11,2015.

[18] Preet Kaur, S. K. aggarwal and Asok De, "Double H Shaped Metamaterial Embedded Compact RMPA", IEEE International conference on advances in computing, communication and informatics (ICACCI), pp. 483-486, September 2014.

[19] Ali A. Saleh, Abdulkareem S. Abdullah, "A Novel Design of Patch Antenna Loaded with Complementary Split-Ring Resonator and L- Shape Slot for (WiMAX/WLAN) Applications," International Journal of Wireless and Microwave Technologies(IJWMT), Vol. 4, No. 3, pp.16-25, October 2014

[20] X. L. Ma, C. Huang, M. B. Pu, W. B. Pan, Y. Q.Wang, and X. G. Luo, "Circular dichroism and optical rotation in twisted Y-shaped chiral metamaterial," Appl. Phys. Expr., vol. 6, p. 022001, 2013. 
[21] M. Mutlu, A. E. Akosman, A. E. Serebryannikov, and E. Ozbay, "Asymmetric chiral metamaterial circular polarizer based on four U-shaped split ring resonators," Opt. Lett., vol. 36, pp. 1653-1655, $2011 .$.

[22] Davoud zarifi, Mohammad Soleimani, Vahid Nayyeri and Jalil Rashed-Mohassel, "On the miniaturization of semi planar chiral metamaterial structures," IEEE Transaction on Antennas and propagation, Vol. 60, No. 12, pp. 5768-5776, dec.2012.

[23] Y.Ye, X.Li, F. Zhuang and S.W.Chang, "Homogenous Circular Polarizer using a bilayered chiral metamaterial," Applied Physics Letter, vol.99, 2011.

[24] J. K. Gansel, M. Thiel, M. S. Rill, M. Decker, K. Bade, V. Saile, G. Freymann, S. Linden, and M. Wegener, "Gold helix photonic metamaterial as broadband circular polarizer," Science, vol. 325, pp. 1513-1515, Sep. 2009.

[25] X. L. Ma, C. Huang, M. Pu, Y. Wang, Z. Zhao, C. Wang, and X. Luo, "Dual-band asymmetry chiral metamaterial based on planar spiral structure," Appl. Phys. Lett., vol. 101, p. 161901, 2012.

[26] J.Zhou, J.Dong,B.Wang, T.Koschny, M.kafesaki and C.M.Soukoulis, "Negative refractive index due to chirality," Phys. Rev. B, Vol. 79,2009.

[27] Davoud zarifi, Mohammad soleimani, Vahid nayyeri, "On the miniaturization of semiplanar choral metamaterial structures," IEEE transaction on Antennas and propagation, Vol.60, No. 12, pp. 5768-5776, December 2012.

[28] B.wang, J.Zhou, T.Koschny, M.Kasesaki and C.M. Soukoulis, "Chiral metamaterials: Simulations and experiments," Journalopt. And pure appl. Opt., vol.11, 2009.

\section{Authors' Profiles}

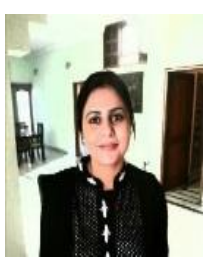

Preet Kaur is B.tech (Electronics \& communication Engg.) from DCRUST, Murthal and M.tech (RF\& Microwave Engg.) from IIT Roorkee and currently pursuing her Ph.D from YMCA University of Science and Technology, Faridabad. Her academic interests include metamaterials, antenna design and electromagnetic wave theory. She is working as Assistant professor (Electronics Engg.) with YMCA University, Faridabad since 2008.

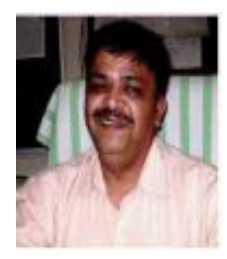

Dr.S.K.Aggarwal is B.tech (Electrical Engg.) from NIT, Calicut (Kerala) and M.tech from Delhi College of Engineering and Ph.D from Jamia Milla Islamiya University, Delhi. He is currently the Professor and Chairman of electronics department with YMCA University of Science and Technology.

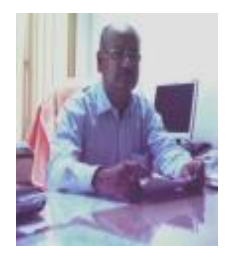

Dr. Asok De received the B.E and M.E degree from Jadavpur university, Kolkata and Ph.D. degrees from IIT Kharagpur in 1986. He is currently the Director of National Institute of Technology, Patna (Bihar). His research interest includes microwave Engg., metamaterials, EM-wave propagation, and antenna miniaturization. 